BMJ Open

Diabetes

Research

\& Care

\section{Lack of association between either outpatient or inpatient glycemic control and COVID-19 illness severity or mortality in patients with diabetes}

Paras B Mehta (D , ${ }^{1}$ Michael A Kohn, ${ }^{2}$ Suneil K Koliwad, ${ }^{1}$ Robert J Rushakoff ${ }^{1}$
To cite: Mehta PB, Kohn MA, Koliwad SK, et al. Lack of association between either outpatient or inpatient glycemic control and COVID-19 illness severity or mortality in patients with diabetes. BMJ Open Diab Res Care 2021;9:e002203. doi:10.1136/ bmjdrc-2021-002203

- Supplemental material is published online only. To view, please visit the journal online (http://dx.doi.org/10.1136/ bmjdrc-2021-002203).

Received 10 February 2021 Accepted 9 May 2021

\section{Check for updates}

\section{(C) Author(s) (or their} employer(s)) 2021. Re-use permitted under CC BY-NC. No commercial re-use. See rights and permissions. Published by BMJ.

\section{${ }^{1}$ Division of Endocrinology} and Metabolism, Department of Medicine, University of California San Francisco, San Francisco, California, USA ${ }^{2}$ Department of Epidemiology and Biostatistics, University of California San Francisco, San Francisco, California, USA

Correspondence to Dr Paras B Mehta; paras.mehta@ucsf.edu

\section{ABSTRACT}

Introduction To evaluate whether outpatient insulin treatment, hemoglobin $\mathrm{A} 1 \mathrm{c}(\mathrm{HbA1C})$, glucose on admission, or glycemic control during hospitalization is associated with SARS-CoV-2 (COVID-19) illness severity or mortality in hospitalized patients with diabetes mellitus (DM) in a geographical region with low COVID-19 prevalence. Research design and methods A single-center retrospective study of patients hospitalized with COVID-19 from January 1 through August 31, 2020 to evaluate whether outpatient insulin use, HbA1c, glucose on admission, or average glucose during admission was associated with intensive care unit (ICU) admission, mechanical ventilation (ventilator) requirement, or mortality.

Results Among 111 patients with DM, 48 (43.2\%) were on outpatient insulin and the average $\mathrm{HbA} 1 \mathrm{C}$ was $8.1 \%$ $(65 \mathrm{mmol} / \mathrm{mol})$. The average glucose on admission was $187.0 \pm 102.94 \mathrm{mg} / \mathrm{dL}$ and the average glucose during hospitalization was $173.4 \pm 39.8 \mathrm{mg} / \mathrm{dL}$. Use of outpatient insulin, level of $\mathrm{HbA} 1 \mathrm{C}$, glucose on admission, or average glucose during hospitalization was not associated with ICU admission, ventilator requirement, or mortality among patients with COVID-19 and DM.

Conclusions Our findings in a region with relatively low COVID-19 prevalence suggest that neither outpatient glycemic control, glucose on admission, or inpatient glycemic control is predictive of illness severity or mortality in patients with DM hospitalized with COVID-19.

\section{INTRODUCTION}

Previous studies have reported poor outcomes for patients with diabetes mellitus (DM) who are hospitalized with SARS-CoV-2 (COVID19) infection. ${ }^{1-8}$ Patients with DM experience longer length of stay, increased risk of severe illness, and higher rates of intensive care unit (ICU) admission, respiratory support, and mortality. ${ }^{1-8}$

While these risks have been widely reported, the effects are variable. Inpatient mortality for large cohorts of patients with DM hospitalized with COVID-19 has ranged from $8 \%$ to $47 \% .^{7-10}$ Yan et $a l^{11}$ reported a mortality of $81 \%$ for patients with DM who had tachypnea, hypoxemia, or severe illness requiring ICU care.

\section{Significance of this study}

What is already known about this subject?

- Limited data, mainly from surge settings, have been published with mixed results on whether worse glycemic control is associated with poor COVID-19 outcomes.

\section{What are the new findings?}

- Outpatient insulin use and hemoglobin A1c were not associated with COVID-19 illness severity or mortality in patients with diabetes in a region with low COVID-19 prevalence.

- Glucose at time of admission was not associated with COVID-19 illness severity or mortality in patients with diabetes in a region with low COVID-19 prevalence.

- Inpatient glycemic control was not associated with COVID-19 illness severity or mortality in patients with diabetes in a region with low COVID-19 prevalence.

How might these results change the focus of research or clinical practice?

- Our study showed that in a non-surge setting with low COVID-19 prevalence, neither outpatient nor inpatient glycemic control was predictive of illness severity or mortality.

- Risk stratification based on pre-existing glycemic control and inpatient glycemic targets may not need to be different for COVID-19 as compared with other illnesses.

Moreover, if DM worsens the severity of COVID-19, it is unclear whether this is a function of glycemic control, or whether either prehospitalization or inpatient glycemic control has any value in predicting COVID-19 disease severity or mortality. Conflicting publications have reported both no effect and worse outcomes for patients with elevated hemoglobin A1c (HbAlc) or who were on insulin treatment prior to hospital admission. ${ }^{912-15}$

Further, heterogeneous definitions and study populations have left the impact of admission glucose and/or inpatient 
hyperglycemia on COVID-19 mortality in patients with DM unclear. ${ }^{8} 10$ 16-21 For example, many studies were conducted using data from the early stages of the pandemic (June 2020 or earlier) in regions experiencing severe community spread (such as New York City, London, and Paris). Both geographical and temporal differences in rates of COVID-19 mortality have arisen since then, and evolution in our understanding of the disease also makes reanalysis of data important. ${ }^{22}$

With this in mind, it is important to study the relationship of DM with COVID-19 outcomes outside of the confines of a large-scale local outbreak. In this setting, intrinsic relationships between DM, glycemic control, and COVID-19, if any, might be better revealed. Based on this, in our region with relatively low COVID-19 hospitalization and mortality during the early months of the pandemic, ${ }^{23}$ we aimed to determine whether outpatient insulin use, HbA1c, glucose at time of admission, or glycemic control during hospitalization is associated with ICU level of care, invasive mechanical ventilation (ventilator) requirement, or mortality.

\section{RESEARCH DESIGN AND METHODS}

Setting and participants

Adult ( $\geq 18$ years of age), non-pregnant patients hospitalized with COVID-19 between January 1, 2020 and August 31, 2020 within the University of California San Francisco (UCSF) Medical Center, a quaternary referral center with three hospitals in the Bay Area of Northern California, were included. As these three hospitals share the same inpatient services, medical providers, administration, and electronic health record (EHR), they were analyzed as a single hospital with three locations.

Patients were further stratified based on a documented diagnosis of DM (as defined in the next section) prior to admission. For patients with DM, inpatient glycemic management at UCSF was consistent with pragmatic recommendations developed by a national expert panel early in the pandemic, which included clustered care, defined as coordinating tasks such as administration of insulin and other medications, meal delivery, and clinical assessment, limiting use of intravenous insulin, aggressive titration of rapid-acting insulin for patients without caloric intake or on enteral feedings, and aggressive adjustment of basal-bolus insulin for patients who are eating. ${ }^{25}$ In addition, all hospitalized patients (irrespective of pre-existing DM) were monitored by our virtual glucose management service (vGMS), and if elevated glucose was found, vGMS notes were placed in the chart suggesting further changes to insulin dosing. ${ }^{26}$

\section{Data collection}

This study was a retrospective review of EHR data. Data were obtained from a combination of automatic extraction using Epic Clarity with predefined variables by a physician with data science experience, as well as detailed chart review of every included patient by one of the authors (PBM).

Demographic and laboratory data were noted. Last HbAlc was defined as the most recent result between 6 months before and 2 weeks into the COVID-19-related hospitalization. DM was defined as either having a documented HbAlc $\geq 6.5 \%$ (48 $\mathrm{mmol} / \mathrm{mol})$, DM listed in outpatient or inpatient diagnoses, and/or DM documented in multiple progress notes during hospitalization. DM diagnoses were reviewed and confirmed during chart review, and no patients were newly diagnosed with DM during the COVID-19-related hospitalization. DM type and outpatient insulin use prior to admission were recorded.

For patients with DM, all measured point-of-care (POC) glucose values during hospitalization were obtained. Consistent with previous glucometric studies, repeat measurements during episodes of hypoglycemia or hyperglycemia were removed to more accurately reflect glycemic control. $^{27} 28$ Mean glucose per patient-day was calculated for each day of hospitalization, and the average glucose level during the entire hospitalization was determined by taking the mean of each of these daily means for a given patient. Additionally, the per cent of days in-range (all blood glucoses 70-180 mg/dL), days with hyperglycemia (two or more glucoses greater than $225 \mathrm{mg} / \mathrm{dL}$ ), and days with hypoglycemia (one or more glucoses less than $70 \mathrm{mg} / \mathrm{dL}$ ) were calculated for each patient with DM. ${ }^{26}$

Patient outcomes included admission to the ICU, ventilator requirement, and mortality.

\section{Statistical analysis}

We report median and IQR for continuous variables, and number and percentage of participants for categorical data. We compared the DM and non-DM groups using the rank-sum test for continuous variables and the exact test for categorical variables. For our outcomes, we report risk ratio with exact $95 \%$ CI. Within the DM group, we calculated the bivariate OR of each glycemic characteristic (outpatient insulin use, HbAlc, admission glucose, mean inpatient glucose) for each of the outcomes (ICU admission, ventilator requirement, mortality). Given the small number of mortality outcomes in the sample, we limited our multivariable logistic regression model to the two binary covariates which had the highest ORs and lowest $\mathrm{p}$ values with respect to our least frequent (mortality) and most frequent (ICU) outcomes. We report multivariable OR with $95 \%$ CI. Stata statistical software was used for all analyses.

Given that our data set encompassed an extended time period, logistic regression was used to evaluate for any changes in ICU admission, ventilator requirement, and mortality over time quartiles for both the DM and non-DM groups. 
Table 1 Baseline characteristics and admission laboratory data of hospitalized patients with COVID-19

\begin{tabular}{|lllc}
\hline Characteristics or laboratory values & $\begin{array}{l}\text { Patients with DM } \\
(\mathbf{n}=111)\end{array}$ & $\begin{array}{l}\text { Patients without DM } \\
(\mathbf{n}=158)\end{array}$ & P value \\
\hline Age $($ years $)$ & $59.2(49.0-71.5)$ & $56.2(42.0-67.1)$ & 0.10 \\
\hline BMI $\left(n^{*}=102, n=139\right)$ & $29.5(25.8-35.1)$ & $26.2(22.4-31.8)$ & $<0.001$ \\
\hline Race & & & 0.20 \\
\hline White & $18(16.2)$ & $42(26.5)$ & \\
\hline Black & $11(9.9)$ & $16(10.1)$ & \\
\hline Latinx & $48(43.2)$ & $55(34.8)$ & \\
\hline Asian & $25(22.5)$ & $26(16.4)$ & 0.44 \\
\hline Other & $9(8.1)$ & $19(12.0)$ & $<0.001$ \\
\hline Male, $n(\%)$ & $67(60.3)$ & $103(65.2)$ & \\
\hline HbA1c $(n=92, n=47)$ & $7.4(6.6-9.3)$ & $5.8(5.5-6.0)$ & $<0.001$ \\
\hline Type 2 DM & $99(89.2)$ & - & 0.85 \\
\hline Outpatient insulin use & $48(43.2)$ & - & 0.38 \\
\hline Glucose $(m g / d L)(n=110, n=150)$ & $163.5(112-225)$ & $108(99-124)$ & 0.04 \\
\hline WBC $\left(\right.$ cells $\left.\times 10^{9} / L\right)(n=111, n=155)$ & $6.8(4.6-8.7)$ & $6.7(4.8-9.4)$ & 0.54 \\
\hline HCO $(m E q / L)(n=111, n=152)$ & $23(21-25)$ & $24(21-26)$ & 0.02 \\
\hline Cr $(m g / d L)(n=110, n=152)$ & $1.1(0.8-1.6)$ & $0.9(0.8-1.2)$ & 0.09 \\
\hline CRP $(m g / L)(n=92, n=127)$ & $65.2(32.5-165.1)$ & $75.3(31.7-125.9)$ & 0.06 \\
\hline Anion gap $(n=110, n=150)$ & $11(9-13)$ & $10(9-12)$ & \\
\hline pH $(n=92, n=122)$ & $7.40(7.35-7.42)$ & $7.41(7.36-7.43)$ & \\
\hline COVID-19 treatment with dexamethasone & $32(43.2)$ & $31(29.8)$ & \\
\hline$(n=74, n=104)$ & & & \\
\hline
\end{tabular}

Data are displayed as median, IQR, or $\mathrm{n}(\%)$.

*When $\mathrm{n}$ is specified in the left column, not all patients had laboratory value obtained.

$\mathrm{BMI}$, body mass index; Cr, creatinine; CRP, C-reactive protein; DM, diabetes mellitus; $\mathrm{HbA1c}$, hemoglobin $\mathrm{A}_{1}$; $\mathrm{HCO}_{3}$, bicarbonate; WBC,

white blood cell count.

\section{RESULTS}

Patient characteristics, admission laboratories, and clinical outcomes

The sample consisted of 269 patients hospitalized with COVID-19, of whom 111 had DM (table 1). The majority of patients were male. Patients with DM had higher baseline body mass index (BMI) than those without DM ( $\mathrm{p}<0.001$ ). The median HbA1c was 7.4\% (IQR 6.6-9.3) in patients with DM and 5.8\% (IQR 5.5-6.0) in patients without DM $(\mathrm{p}<0.001)$. At admission, patients with DM had higher serum glucose values and serum creatinine
(Cr) concentrations and a wider anion gap ( $\mathrm{p}<0.05$ for each laboratory measurement). Serum white blood cell count (WBC), bicarbonate $\left(\mathrm{HCO}_{3}\right)$, C-reactive protein (CRP), and $\mathrm{pH}$ were similar between the groups. Of patients admitted after dexamethasone treatment was offered for COVID-19 at our institution, $43.2 \%$ with $\mathrm{DM}$ and $29.8 \%$ without DM received dexamethasone $(\mathrm{p}=0.06)$, respectively.

Of the 269 patients, 117 (43.9\%) required ICU admission, $70(26.0 \%)$ required ventilator support, and 16 $(5.9 \%)$ died in the hospital (table 2). Patients with DM

Table 2 Outcomes in hospitalized patients with COVID-19

\begin{tabular}{llllll}
\hline \multicolumn{7}{c}{ Characteristics } & $\begin{array}{l}\text { Patients with DM } \\
(\mathbf{n = 1 1 1 )}\end{array}$ & $\begin{array}{l}\text { Patients without } \\
\mathbf{D M} \\
\mathbf{( n = 1 5 8 )}\end{array}$ & $\begin{array}{l}\text { Relative risk } \\
\mathbf{( 9 5 \% ~ C l )}\end{array}$ & $\begin{array}{l}\text { Difference, } \\
\text { \% (95\% Cl) }\end{array}$ & P value \\
\hline Length of stay (days) & $12.0(5.5-22.5)$ & $8.0(4.0-16.0)$ & $1.39(1.06$ to 1.82$)$ & $2.2(0.5$ to 4.1$)$ & 0.01 \\
\hline ICU care & $52(46.8)$ & $65(41.1)$ & $1.14(0.87$ to 1.49$)$ & $5.7(-6.3$ to 17.8$)$ & 0.38 \\
\hline Ventilator support & $34(30.6)$ & $36(22.8)$ & $1.34(0.90$ to 2.00$)$ & $7.8(-2.9$ to 18.6$)$ & 0.16 \\
\hline Mortality & $8(7.2)$ & $8(5.1)$ & $1.42(0.55$ to 3.68$)$ & $2.1(-3.7$ to 8.0$)$ & 0.60 \\
\hline
\end{tabular}

Data are displayed as median, IQR, or $\mathrm{n}(\%)$.

DM, diabetes mellitus; ICU, intensive care unit. 
were more likely to require ICU admission (relative risk (RR) 1.14), ventilator support (RR 1.34), and die in the hospital (RR 1.42) than those without DM, but the differences were not statistically significant.

There were no statistically significant changes in ICU admission, ventilator requirement, or mortality over time in both the DM and non-DM groups.

\section{Association of glycemic control with clinical outcomes}

Of the 111 patients with DM, 92 (83\%) had an HbAlc within the specified timeframe and 110 (99\%) had admission serum glucose checked to be included in each respective analysis. Ninety-six $(86 \%)$ had POC glucose data collected during hospitalization and were included in the inpatient glycemic control analysis; the remainder of the patients either had brief hospitalizations or wellcontrolled serum glucoses which precluded the need for POC testing. The number of POC glucose readings per patient, per hospitalization, ranged from 3 to 1354, with a median of 58 and IQR of 25-134. The mean POC glucose per patient-day for these 96 patients was $173.4 \pm 39.8$ $\mathrm{mg} / \mathrm{dL}$. As defined in the prior section, blood glucose remained in-range during $30.8 \%$ of patient-days. Patients were hyperglycemic during $25.3 \%$ of days and hypoglycemic during $4.4 \%$ of days.

Bivariate analysis revealed age $\geq 75$ years and male sex to be most strongly associated with the outcomes (online supplemental appendix). These two variables were included in our multivariable model. Given that neither BMI nor creatinine was associated with the outcomes $(p>0.05)$, these variables were not included. Table 3 depicts the unadjusted (bivariate) and adjusted (multivariable) ORs for patients with DM. In both unadjusted and adjusted analyses, the associations of the independent variables (outpatient insulin use, HbAlc, admission serum glucose, and mean glucose per patient-day during hospitalization) and the outcomes (ICU care requirement, ventilator requirement, and mortality) were variable and not statistically significant. For example, outpatient use of insulin had an adjusted OR for ventilator requirement of 1.71 but for in-hospital death of 0.71 .

The analysis for serum glucose on admission was repeated for all included patients irrespective of DM. For this analysis, 260 (97\%) patients had admission serum glucose available for review. In adjusted analyses, higher admission glucose was associated with ICU care requirement (OR 1.04, 95\% CI 1.00 to 1.07 ) and ventilator requirement (OR 1.05, 95\% CI 1.02 to 1.09 ), but not mortality (OR $1.02,95 \%$ CI 0.97 to 1.08 ).

\section{CONCLUSIONS}

For patients with DM hospitalized with COVID-19, we found no consistent association between outpatient insulin use, HbA1c, serum glucose on admission, or average inpatient glucose with ICU admission, ventilator requirement, or mortality. While there was higher mortality in patients with DM compared with patients without DM, the difference was not statistically significant. Our observed mortality for patients with DM of $7.2 \%$ is 
notably lower than prior studies in similar patients which have reported mortalities more than 25\%.9 ${ }^{1529}$ This low COVID-19 mortality is consistent with recent findings that outcomes improved when, as observed in San Francisco, community prevalence of COVID-19 was lower and hospitals had fewer cases. ${ }^{22-24}$ We posit that this is due to hospital systems maintaining adequate resources to provide high level of care rather than COVID-19 illness being less severe in regions with low prevalence.

The mean and median $\mathrm{HbAlc}$ values for patients with DM were $8.1 \%(65 \mathrm{mmol} / \mathrm{mol})$ and $7.4 \%(57 \mathrm{mmol} /$ $\mathrm{mol})$, respectively, indicating that many did not meet outpatient glycemic targets. Despite this variable outpatient control, we found no difference in outcomes based on prehospitalization HbAlc, suggesting pre-existing glycemic control may not be useful for risk stratification. This lack of association with HbAlc and illness severity is in line with multiple other studies of hospitalized patients with COVID-19. ${ }^{9} 1114$ However, we do note that some studies have noted a possible link between HbAlc and illness severity. In part, this difference may be attributed to methodological variation. Zhu et al found that HbAlc was an independent risk factor for poor outcomes, but used $\mathrm{HbAlc} \geq 6.5 \%(48 \mathrm{mmol} / \mathrm{mol})$ as the criterion for insufficient glycemic control. ${ }^{10}$ By using this cut-off, which is misaligned with accepted society guidelines, patients with adequate outpatient glycemic control were potentially under-represented. ${ }^{30}$ A population-based data registry analysis from England indicated that an HbAlc $\geq 7.6 \%(60 \mathrm{mmol} / \mathrm{mol})$ in patients with type $2 \mathrm{DM}$ was associated with increased mortality from COVID-19. ${ }^{13}$ These data included many ambulatory patients and did not specifically address outcomes of hospitalized patients.

We also found no association between outpatient insulin use and illness severity or mortality. Others have reported variable associations of insulin use and mortality. ${ }^{9} 111415$ Cariou $e t ~ a l^{14}$ showed that outpatient insulin use was not associated with the composite outcome of ventilator requirement and mortality. To our knowledge, our study is the first report on the association of outpatient insulin use and ICU admission specifically in COVID-19.

Similarly, we found no association with admission serum glucose and any of the outcomes in patients with DM. We did however find that admission glucose was associated with increased ICU admission and ventilator requirement when evaluated across all patients in adjusted analyses, possibly indicating a role of stress hyperglycemia in patients without DM. This finding across all patients is in line with other published data, as an association with higher admission glucose values and ICU care, ventilator requirement, and mortality has been found. ${ }^{14}$ 17-19

In terms of inpatient glycemic control, we found no association with ICU admission, ventilator requirement, or mortality. Our patient group with DM represents a spectrum of illness severity and included both critically ill and non-critically ill patients. The overall mean glucose per patient-day $(173.4 \pm 39.8 \mathrm{mg} / \mathrm{dL})$ was near the upper end of the target $140-180 \mathrm{mg} / \mathrm{dL}$ range recommended for most ICU and non-ICU hospitalized patients, indicating that there may not be significant benefit to targeting any tighter glycemic control in these patients. ${ }^{31-33}$

Others have reported an association with inpatient hyperglycemia and worse outcomes in COVID-19, but these studies have differences from ours, including limited analysis on patient subgroups with DM, varying definitions of hyperglycemia (eg, $140 \mathrm{mg} / \mathrm{dL}$ vs $180 \mathrm{mg}$ / dL vs using 2-hour postprandial glucose), and varying patient populations (eg, exclusion of non-critically ill patients or inclusion of all patients irrespective of diabetes status). ${ }^{8101619}$ The overall paucity and heterogeneity in the data highlight an important area where continued research is needed given the known implications of glycemic control in patients hospitalized with other medical conditions.

Our data reflect a time interval during which case counts and medical resources varied, and scientific understanding of COVID-19 rapidly evolved, whereas other studies related to glycemic control were performed during the initial surge of the pandemic..$^{912-14}$ We also included patients at all levels of inpatient care (acute care, intermediate care, and ICU), whereas other data have focused on critically ill patients or included ambulatory patients within a population-based registry. ${ }^{12} 1316$ We hope this patient population may be representative of future COVID-19 hospitalizations, as we know that COVID-19 outcomes improved in nearly all hospitals in the USA over the first 6 months of the pandemic. ${ }^{22}$

Our study has several limitations. This was a singlecenter study at a quaternary referral center, and it is possible our patient population may be different in complexity from that of other hospitals. The low mortality rate may also have limited detection of certain trends, although we believe these data are more representative of hospitalized patients with COVID-19 moving forward. We would also like to highlight an inherent limitation of studies based on automated electronic data extractionwhen we performed a manual chart review on these 269 patients, we noted inaccuracies with extraction of DM diagnosis and missing laboratory and prescription data from medical care outside of our health system. We were able to manually verify the data on every patient in this study, but this degree of data validation may be challenging in data sets with thousands of patients.

Given the differing results among currently published data, and the geographical and temporal variations in COVID-19 prevalence and mortality, continued data collection is needed to evaluate the role of glycemic control. Ongoing multicenter studies may help clarify these issues.

In summary, we showed that in a setting with low COVID-19 prevalence and mortality, outpatient glycemic control, as indicated by use of insulin therapy and HbAlc, is not associated with illness severity or mortality in hospitalized patients with COVID-19 with DM. Additionally, neither admission serum glucose nor subsequent inpatient glycemic control is associated with illness severity or mortality. These results indicate that these glycemic 
measures should not be used for risk stratification in COVID-19, and that glycemic management for patients with COVID-19 with DM should be approached similarly to accepted inpatient guidelines.

Acknowledgements We would like to acknowledge Logan Pierce, MD and Nader Najafi, MD of the Division of Hospital Medicine, University of California, San Francisco for their assistance in extracting initial data for our study.

Contributors PBM analyzed the data, performed the literature review, and drafted the manuscript. MAK assisted with statistical analysis and manuscript revision. SKK assisted with manuscript revision and literature review. RJR assisted with research design, manuscript writing, manuscript revision, and literature review.

Funding This work was supported by the National Institutes of Health (T32DK007418).

\section{Competing interests None declared.}

Patient consent for publication Not required.

Ethics approval This research project was approved by the University of California, San Francisco's Institutional Review Board and was deemed minimal risk to human subjects. The approval ID number is 20-32006.

Provenance and peer review Not commissioned; externally peer reviewed.

Data availability statement Data are available upon reasonable request. Data are stored on a secure electronic server at the University of California, San Francisco.

Supplemental material This content has been supplied by the author(s). It has not been vetted by BMJ Publishing Group Limited (BMJ) and may not have been peer-reviewed. Any opinions or recommendations discussed are solely those of the author(s) and are not endorsed by BMJ. BMJ disclaims all liability and responsibility arising from any reliance placed on the content. Where the content includes any translated material, BMJ does not warrant the accuracy and reliability of the translations (including but not limited to local regulations, clinical guidelines, terminology, drug names and drug dosages), and is not responsible for any error and/or omissions arising from translation and adaptation or otherwise.

Open access This is an open access article distributed in accordance with the Creative Commons Attribution Non Commercial (CC BY-NC 4.0) license, which permits others to distribute, remix, adapt, build upon this work non-commercially, and license their derivative works on different terms, provided the original work is properly cited, appropriate credit is given, any changes made indicated, and the use is non-commercial. See: http://creativecommons.org/licenses/by-nc/4.0/.

ORCID iD

Paras B Mehta http://orcid.org/0000-0001-5434-5424

\section{REFERENCES}

1 Kumar A, Arora A, Sharma P, et al. Is diabetes mellitus associated with mortality and severity of COVID-19? A meta-analysis. Diabetes Metab Syndr 2020;14:535-45.

2 loannou GN, Locke E, Green P, et al. Risk Factors for Hospitalization, Mechanical Ventilation, or Death Among 10131 US Veterans With SARS-CoV-2 Infection. JAMA Netw Open 2020;3:e2022310.

3 Fox T, Ruddiman K, KB L. The relationship between diabetes and clinical outcomes in COVID-19: a single-center retrospective analysis. Acta Diabetol 2020:1-6.

4 Guan WJ, ZY N, Hu Y. China medical treatment expert group for Covid-19. clinical characteristics of coronavirus disease 2019 in China. N Engl J Med 2020;382:1708-20.

5 Targher G, Mantovani A, Wang X-B, et al. Patients with diabetes are at higher risk for severe illness from COVID-19. Diabetes Metab 2020;46:335-7.

6 Guo W, Li M, Dong Y, et al. Diabetes is a risk factor for the progression and prognosis of COVID-19. Diabetes Metab Res Rev 2020:e3319.

7 Zhou F, Yu T, Du R, et al. Clinical course and risk factors for mortality of adult inpatients with COVID-19 in Wuhan, China: a retrospective cohort study. Lancet 2020;395:1054-62.

8 Bode B, Garrett V, Messler J, et al. Glycemic characteristics and clinical outcomes of COVID-19 patients hospitalized in the United States. J Diabetes Sci Technol 2020;14:813-21.

9 Agarwal S, Schechter C, Southern W, et al. Preadmission Diabetes-Specific risk factors for mortality in hospitalized patients with diabetes and coronavirus disease 2019. Diabetes Care 2020;43:2339-44.
10 Zhu L, She Z-G, Cheng X, et al. Association of blood glucose control and outcomes in patients with COVID-19 and pre-existing type 2 diabetes. Cell Metab 2020;31:1068-77.

11 Yan Y, Yang Y, Wang F, et al. Clinical characteristics and outcomes of patients with severe covid-19 with diabetes. BMJ Open Diabetes Res Care 2020;8:e001343.

12 Liu L, Wei W, Yang K, et al. Glycemic control before admission is an important determinant of prognosis in patients with coronavirus disease 2019. J Diabetes Investig 2020. doi:10.1111/jdi.13431. [Epub ahead of print: 09 Oct 2020].

13 Holman N, Knighton P, Kar P, et al. Risk factors for COVID-19-related mortality in people with type 1 and type 2 diabetes in England: a population-based cohort study. Lancet Diabetes Endocrinol 2020;8:823-33.

14 Cariou B, Hadjadj S, Wargny M, et al. Phenotypic characteristics and prognosis of inpatients with COVID-19 and diabetes: the CORONADO study. Diabetologia 2020;63:1500-15.

15 Sourij H, Aziz F, Bräuer A, et al. COVID-19 fatality prediction in people with diabetes and prediabetes using a simple score upon hospital admission. Diabetes Obes Metab 2021;23:589-598.

16 Saand AR, Flores M, Kewan T. Does Inpatient Hyperglycemia predict a worse outcome in COVID-19 Intensive Care Unit Patients? [published online ahead of print, 2020 Nov 20].. J Diabetes 2020.

17 Carrasco-Sánchez FJ, López-Carmona Mª Dolores, MartínezMarcos FJ, et al. Admission hyperglycaemia as a predictor of mortality in patients hospitalized with COVID-19 regardless of diabetes status: data from the Spanish SEMI-COVID-19 registry. Ann Med 2021:53:103-16.

18 Sardu C, D'Onofrio N, Balestrieri ML, et al. Outcomes in patients with hyperglycemia affected by COVID-19: can we do more on glycemic control? Diabetes Care 2020;43:1408-15.

19 Klonoff DC, Messler JC, Umpierrez GE, et al. Association between achieving inpatient glycemic control and clinical outcomes in hospitalized patients with COVID-19: a multicenter, retrospective hospital-based analysis. Diabetes Care 2021;44:dc201857.

20 Chen L, Sun W, Liu Y, et al. Association of early-phase in-hospital glycemic fluctuation with mortality in adult patients with coronavirus disease 2019. Diabetes Care 2021;44:865-73.

21 Shen Y, Fan X, Zhang L, et al. Thresholds of glycemia and the outcomes of COVID-19 complicated with diabetes: a retrospective exploratory study using continuous glucose monitoring. Diabetes Care 2021;44:976-82.

22 Asch DA, Sheils NE, Islam MN. Variation in US Hospital Mortality Rates for Patients Admitted With COVID-19 During the First 6 Months of the Pandemic [published online ahead of print, 2020 Dec 22].. JAMA Intern Med 2020.

23 Johns Hopkins Coronavirus Resource Center. Johns Hopkins University, 2020. Available: https://coronavirus.jhu.edu/ [Accessed 21 Nov 2020].

24 City and County of San Francisco. San Francisco COVID-19 data and reports, 2020. Available: https://data.sfgov.org/stories/s/fjki-2fab [Accessed 21 Nov 2020].

25 Korytkowski M, Antinori-Lent K, Drincic A, et al. A pragmatic approach to inpatient diabetes management during the COVID-19 pandemic. J Clin Endocrinol Metab 2020;105:3076-87.

26 Rushakoff RJ, Sullivan MM, MacMaster HW, et al. Association between a virtual glucose management service and glycemic control in hospitalized adult patients: an observational study. Ann Intern Med 2017;166:621-7.

27 Weinberg ME, Bacchetti P, Rushakoff RJ. Frequently repeated glucose measurements overestimate the incidence of inpatient hypoglycemia and severe hyperglycemia. J Diabetes Sci Technol 2010;4:577-82.

28 Goldberg PA, Bozzo JE, Thomas PG, et al. "Glucometrics"-assessing the quality of inpatient glucose management. Diabetes Technol Ther 2006;8:560-9.

29 Mansour A, Sajjadi-Jazi SM, Kasaeian A, et al. Clinical characteristics and outcomes of diabetics hospitalized for COVID-19 infection: a single-centered, retrospective, observational study. Excli J 2020;19:1533-43.

30 American Diabetes Association. 6. Glycemic Targets: Standards of Medical Care in Diabetes-2020. Diabetes Care 2020;43:S66-76.

31 American Diabetes Association. 15. Diabetes Care in the Hospital: standards. Diabetes Care 2019;42:S173-81.

32 NICE-SUGAR Study Investigators, Finfer S, Chittock DR, et al. Intensive versus conventional glucose control in critically ill patients. N Engl J Med 2009;360:1283-97.

33 Umpierrez GE, Hellman R, Korytkowski MT, et al. Management of hyperglycemia in hospitalized patients in non-critical care setting: an endocrine Society clinical practice guideline. J Clin Endocrinol Metab 2012;97:16-38. 